\title{
Performance Study of Series Loaded Resonant Converter Using Super Barrier Rectifiers
}

\author{
Taufik Taufik; Michael McCarthy; Scott Watkins \\ Electrical Engineering Department \\ California Polytechnic State University \\ San Luis Obispo, California, USA
}

\author{
Makbul Anwari \\ Faculty of Electrical Engineering \\ Universiti Teknologi Malaysia \\ 81310 UTM Skudai, Malaysia
}

\begin{abstract}
In power electronics efficiency improvement is constantly being sought out through venues such as improved topologies or improved devices. Series Loaded Resonant (SLR) DC-DC converter is a type of soft-switching topology widely known for providing improved efficiency. This paper investigates the efficiency and transient performance of SLR when a new type of diode called Super Barrier Rectifier ${ }^{\mathrm{TM}}$ is being used. The design of SLR, results from computer simulations, and hardware measurements will be discussed. The efficiency measurements from all conduction modes in SLR using three types of diodes (pn, schottky, and SBR) will be presented along with transient study of one of SLR's continuous conduction mode.
\end{abstract}

Keywords - Series Loaded Resonant, Super Barrier Rectifier Diodes

\section{INTRODUCTION}

One major branch of power electronics is in the area of DC to DC converters. DC-DC converters change an input voltage to a different output voltage as efficiently as possible. In general, these dc-dc converters are operated with square-wave switching (hard-switching) and softswitching. The soft-switching topologies are known to be more complex than their equivalent hard-switching topology, but they can provide improved converter's efficiency. An example of a soft-switching topology is the Series Loaded Resonant (SLR) shown in Figure 1.

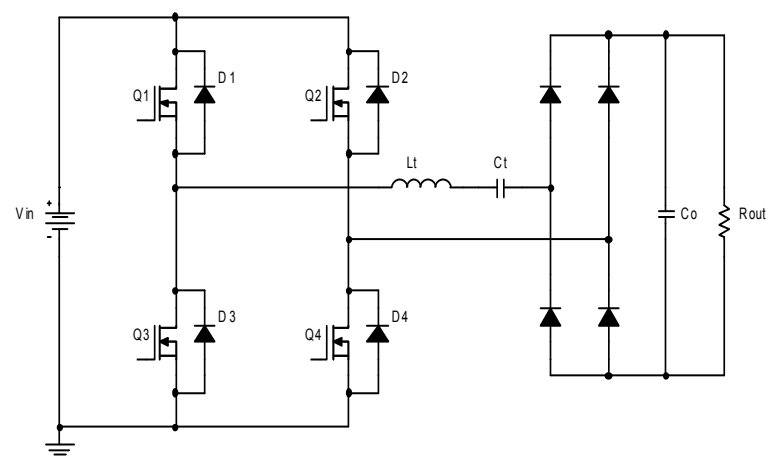

Figure 1. Pspice schematic for the SLR Converter
DC-DC converters operate by switching the input voltage on and off at high frequencies. When a switch turns on (off) it takes a small amount of time for its voltage (current) to drop to zero. During this time converter experiences switching losses. Hard switching occurs when the switch is turned on (off) when the voltage (current) is non-zero. When the voltage or current within the converter is switched as the voltage or current is naturally zero, softswitching is said to occur.

Loaded resonant converters are converters that utilize the soft-switching techniques. A loaded resonant converter works by inverting a DC input into an $\mathrm{AC}$ waveforms and rectifying the $\mathrm{AC}$ waveform back into a $\mathrm{DC}$ signal. The circuit consists of a bridge converter, a resonant tank, a rectifier, and an output filter. The resonant tank, which consists of an inductor and capacitor, can be configured in many ways including parallel, series, or both parallel and series (hybrid). In all of these converters the current naturally commutates to zero limiting switching losses. Hence, by timing the switching signals carefully, the converters may provide improved efficiency.

The series loaded resonant (SLR) converter has three modes of operation that are determined by the switching frequencies relationship with the resonant frequency of the resonant tank. The Discontinuous Conduction Mode (DCM) occurs when the switching frequency is less than half of the resonant frequency, whereas Continuous Conduction Mode below resonance (CCM 1) occurs when the switching frequency ranges from $50 \%$ to $100 \%$ of the resonant frequency, and CCM above resonance (CCM 2) occurs when the switching frequency is greater than the resonant frequency. A switch is said to have zero-voltage switching (ZVS) and zero-current switching (ZCS) when the voltage/current is zero as the switch changes states. A summary of SLR switching properties is listed in Table 1.

While there have been many published performance studies of resonant converters as in [1][2][3][4], none has specifically investigated the effect of the diode's impact on the converter's performance. The focus of this paper is therefore to compare the efficiency performance of the SLR converter with three different types of diodes: the Schottky diode, $\mathrm{p}-\mathrm{n}$ diode, and the Super Barrier Recitifier ${ }^{\mathrm{TM}}$ (SBR). 
In addition, a transient performance of SLR using the three diodes will also be presented. However, as indicated in Table 1, since CCM 1 is the only mode that requires a fastrecovery diode, therefore the transient study could only be applied to CCM 1. Reverse recovery is the phenomenon when a diode is turned-off (occurs at turn-on of the switch) it takes a certain amount of time $\left(t_{\mathrm{rr}}\right)$ before it can block reverse voltages. This is a result of the excess carriers in the diode that need to be swept out [6]. This limits the frequency at which the converter can be operated and using a fast recovery diode allows for faster reverse recovery and hence increased frequency.

Table 1. Summary of SLR Switching Properties

\begin{tabular}{clll} 
& Switch Transition & ZCS & ZVS \\
\hline & Turn-on & Yes & No \\
DCM & Turn-off & Yes & Yes \\
\hline & Turn-on & No & No \\
CCM1 & Turn-off & Yes & Yes \\
\hline & Turn-on & Yes & Yes \\
CCM2 & Turn-off & No & No \\
\hline
\end{tabular}

The three different diodes used have their design tradeoffs. The Schottky diode has a fast reverse recovery time; however, they typically have a large reverse leakage current that contributes to conduction losses. The $\mathrm{p}-\mathrm{n}$ diodes usually have a lower reverse leakage current, but their reverse recovery time is much larger as is their forward voltage drop contributing to conduction losses outside of switching. The SBR combines the beneficial characteristics of both other types [6]. They have a low forward voltage drop and reverse leakage current and their reverse recovery time is comparable to Schottky diodes. The recovery is also much smoother limiting the ringing and other oscillations that can occur throughout the circuit.

This study will focus on the performance of the SLR converter specifically in two areas--the overall efficiency of the converter and its switching transients. A short summary of the SLR converter design will be described next, followed by computer simulations using OrCAD Pspice. To further verify these two areas a laboratory setup will be used to obtain actual hardware measurements.

\section{DESIGN SUMMARY}

The design of the SLR converter focused on the performance of the anti-parallel diodes, resonant frequency, component stresses, and transistor losses. The parameters were chosen in order to test the converter's operation in CCM 1.

The MOSFET was chosen as a Fairchild FDP6030BL because of its low drain-to-source resistance. Since its maximum drain-to-source voltage was $30 \mathrm{~V}$ the input was limited to this voltage. In order to obtain a resonant frequency of $90 \mathrm{kHz}$ and characteristic impedance of $50 \Omega$, a $33 \mathrm{nF}$ capacitor was chosen and a $94.7 \mathrm{uH}$ inductor was wound. The equations for the inductor current and capacitor voltage can be shown as:

$$
\begin{gathered}
i_{L}(t)=1.13 \sin \left(\omega_{0} t\right) \text { and } i_{L-\max }=1.13 \mathrm{~A} \\
V_{C}(t)=30-60 \cos \left(\omega_{0} t\right) \text { and } V_{C-\max }=90 \mathrm{~V}
\end{gathered}
$$

The diodes were chosen based on peak current conditions and a blocking ability of at least $30 \mathrm{~V}$. Their electrical characteristics are shown in Table 2. The selected SBR actually has a higher reverse leakage current than the Schottky diode and the reverse recovery time is slightly higher than the $\mathrm{p}-\mathrm{n}$ diode, but still comparable to the Schottky. Also, it is estimated that the SBR has a high amount of junction capacitance, although the exact amount is not specified in the datasheet. Reverse recovery time for Schottky diode is estimated from typical values.

Table 2. Electrical Characteristics of the Diodes Being Compared in the

\begin{tabular}{|c|c|c|c|}
\hline Electrical Characteristics & $\begin{array}{l}\text { Schottky } \\
\text { MBR1045 }\end{array}$ & $\begin{array}{l}\text { P-N Diode } \\
\text { STPR1020CT }\end{array}$ & $\begin{array}{l}\text { SBR } \\
\text { SBR10U } \\
40 \mathrm{CT}\end{array}$ \\
\hline Vf, typical $\left(\mathrm{I}_{\mathrm{f}}=10 \mathrm{~A}\right)$ & $0.61 \mathrm{~V}$ & $<0.95 \mathrm{~V}$ & $0.47 \mathrm{~V}$ \\
\hline $\mathrm{Vf}, \operatorname{maximum}\left(\mathrm{I}_{\mathrm{f}}=10 \mathrm{~A}\right)$ & $0.64 \mathrm{~V}$ & $1.25 \mathrm{~V}$ & $0.52 \mathrm{~V}$ \\
\hline $\begin{array}{l}\text { Reverse leakage current, } \\
\text { typical }\end{array}$ & $0.008 \mathrm{~mA}$ & not provided & $0.15 \mathrm{~mA}$ \\
\hline $\begin{array}{l}\text { Reverse leakage current, } \\
\text { maximum }\end{array}$ & $0.1 \mathrm{~mA}$ & $0.05 \mathrm{~mA}$ & $0.5 \mathrm{~mA}$ \\
\hline Junction Capacitance, $\mathrm{C}_{\mathrm{i}}$ & $\begin{array}{l}150-1000 \\
\mathrm{pF}\end{array}$ & $15-25 \mathrm{pF}$ & $\begin{array}{l}>150- \\
1000 \mathrm{pF}\end{array}$ \\
\hline Reverse recovery time, $t_{\mathrm{rr}}$ & $\sim 20 \mathrm{~ns}$ & $30 \mathrm{~ns}$ & $32 \mathrm{~ns}$ \\
\hline
\end{tabular}
Current Study

The resonant frequency was chosen and experimentally measured to be around $85.9 \mathrm{kHz}$. It is important to know at what switching frequency each mode of operation will occur. Based on the selected resonant frequency, the DCM will be operated at a switching frequency less than $43 \mathrm{kHz}$, while for CCM 1 it will range from $43 \mathrm{kHz}$ to $85.9 \mathrm{kHz}$, and for CCM 2 it will be above $85.9 \mathrm{kHz}$.

\section{COMPUTER SIMULATION}

Following the design and prior to building the hardware for lab testing, a computer simulation was conducted using OrCAD PSpice. Figure 2 depicts the schematic used in simulating the SLR converter. 


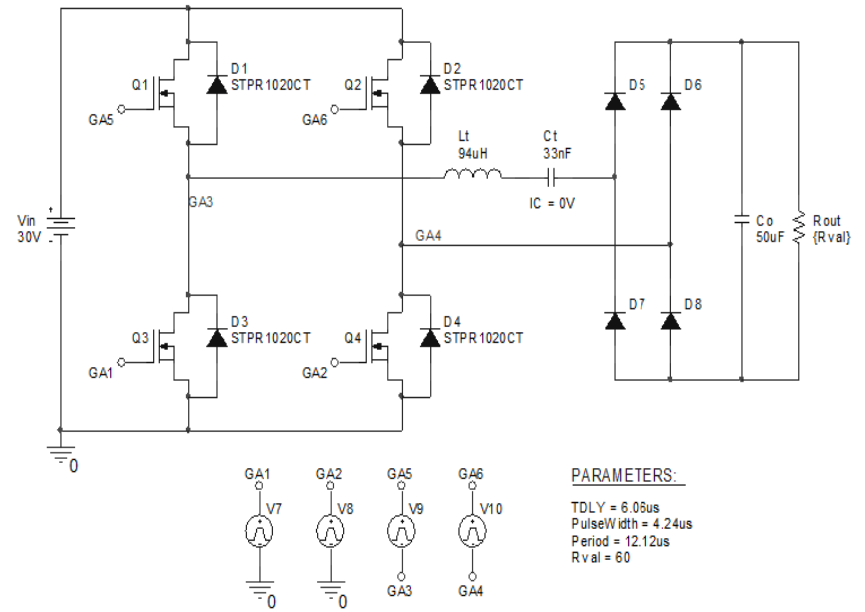

Figure 2. Pspice schematic for the SLR Converter

The Model Editor within the OrCAD PSpice software was used to model the reverse recovery characteristics of the three diodes as shown in Figure 3. The simulated reverse recovery characteristics for the three diodes are illustrated in Figure 3.
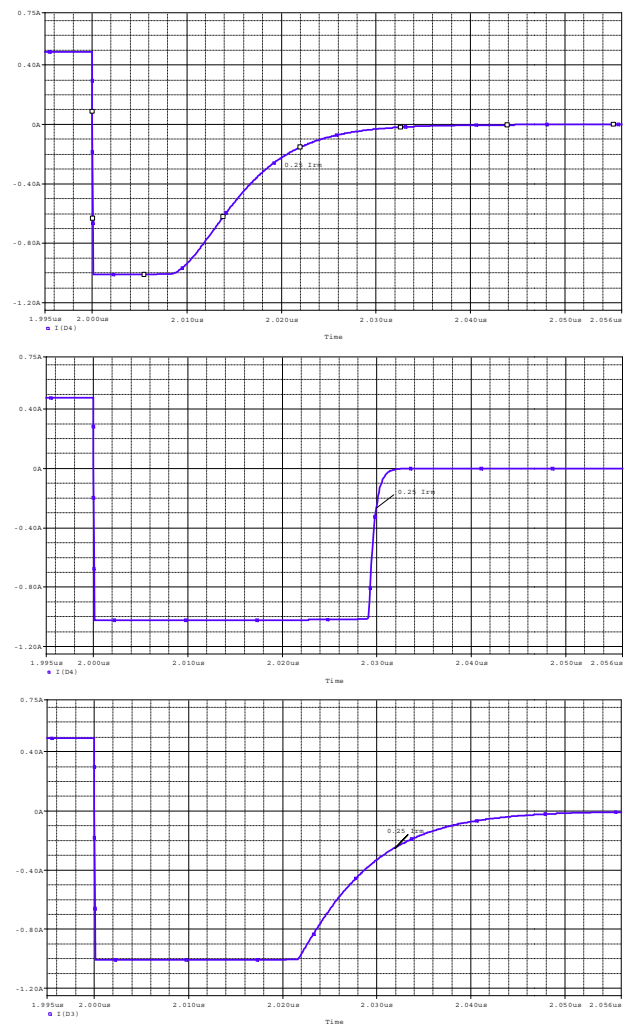

Figure 3. Simulated reverse recovery characteristics for schottky (top), pn (middle), and SBR (bottom).

Figure 4 shows an example of an efficiency plot obtained while the converter is operated in CCM 1. A switching frequency of $82.5 \mathrm{kHz}$ was used to ensure converter operation in CCM 1. The load was varied using the parametric sweep function allowing the efficiency to be measured over a range of output power. As evidenced in Figure 4, the p-n diode outperformed both Schottky and SBR at lower loads. This is expected since the $\mathrm{p}$-n diode has the lowest reverse leakage current. However, as the power level increases $p$-n diode's performance decreases. This is because at higher load (thus, higher frequencies) more switching occurs and the reverse recovery time plays a more important role. This is why Schottky has the best performance at high power levels. The SBR on the other hand behaves like the Schottky at low power because of its higher reverse leakage current but behaves more like a p-n diode at high power because of its similar reverse recovery time.

To demonstrate the different conduction modes, Figure 5 is provided which shows the resonant inductor current waveform in each type of conduction mode.



Figure 4. Converter Efficiency vs. Output Power for three diode cases
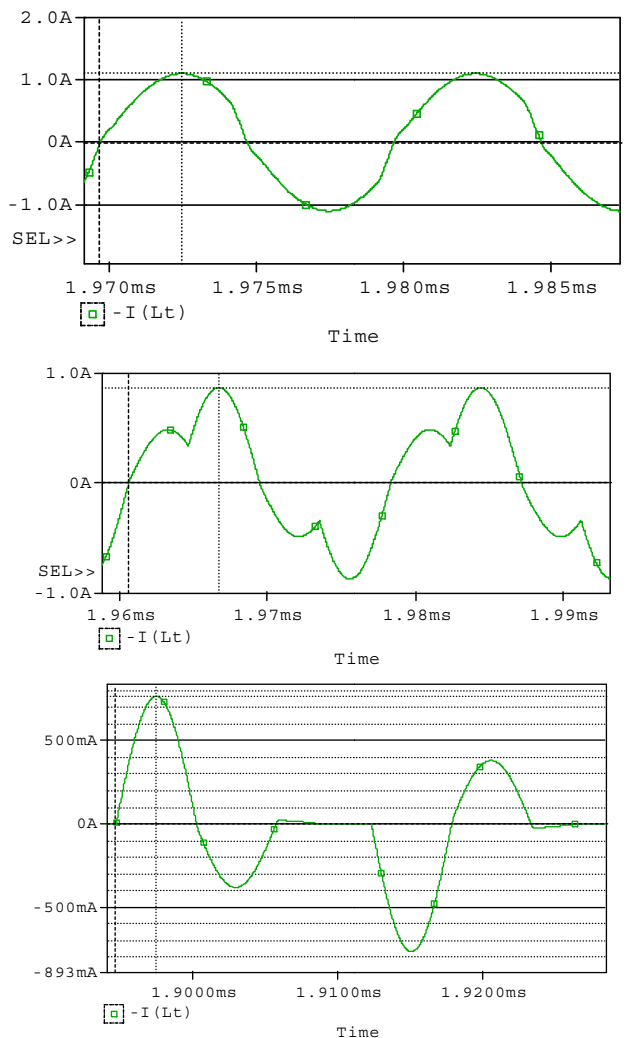

Figure 5. Resonant inductor current waveform in CCM 1 (top), CCM 2 (middle), and DCM (bottom) 


\section{HARDWARE MEASUREMENT}

The circuit of Figure 2 was built on a proto-board as shown in Figure 6. Wire and lead lengths were minimized to reduce the amount of stray inductance between elements. Although it was not completely avoidable the stray inductance present did not add up to a significant amount of loss.

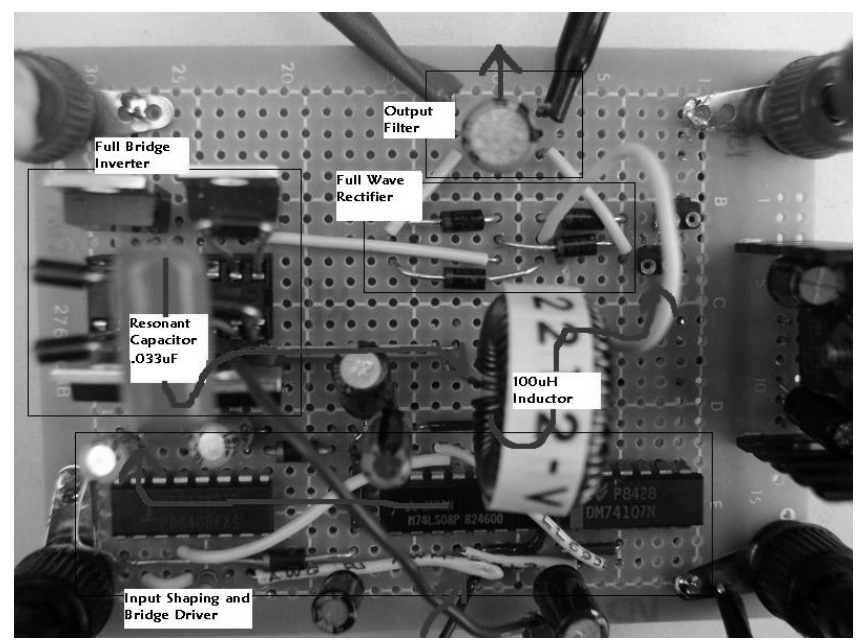

Figure 6. The SLR Circuit

Figure 7 exhibits the resonant inductor current waveforms in all three modes. The waveforms show close resemblance with those shown in Figure 5 for each corresponding conduction mode.

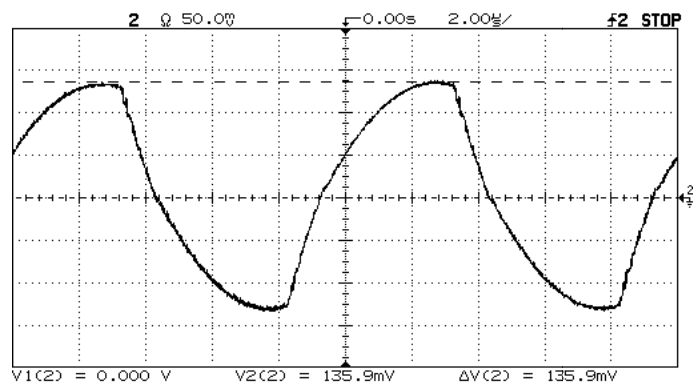

(a)

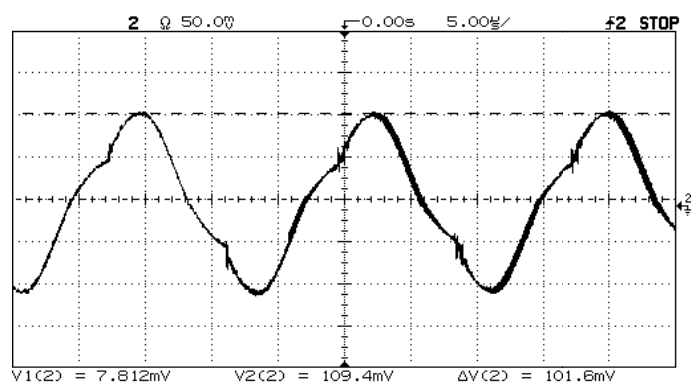

(b)

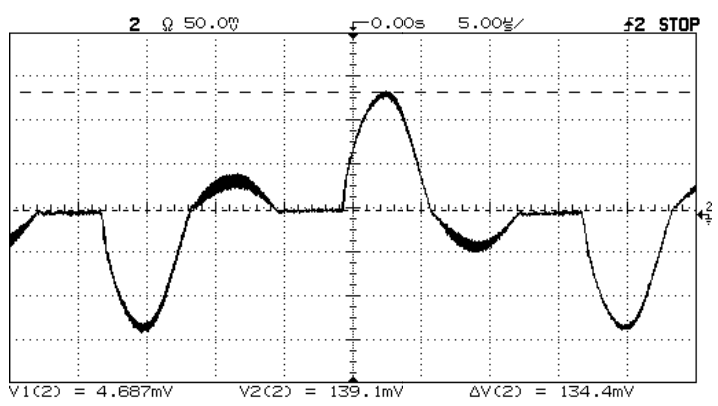

(c)

Figure 7. Resonant inductor current waveform in CCM 1 (a), CCM 2 (b), and DCM (c)
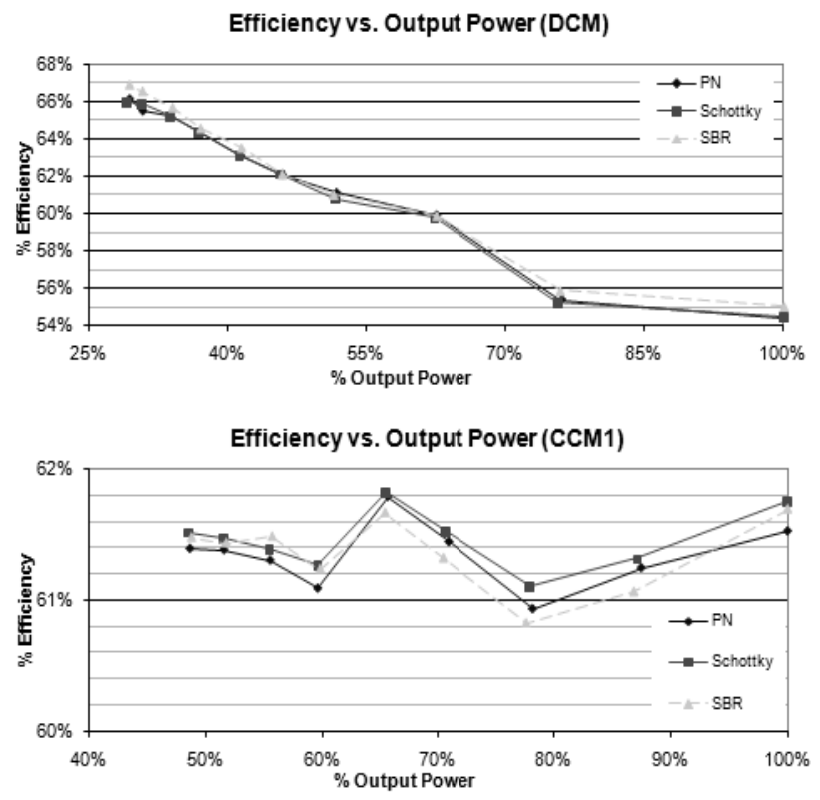

Efficiency vs. Output Power (CCM2)

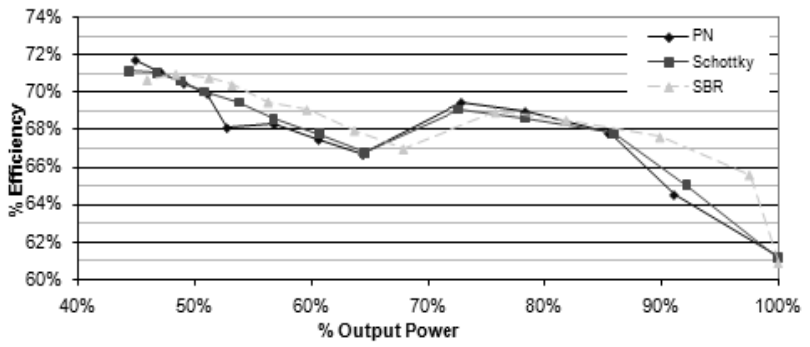

Figure 8. Efficiency vs. Output Power for DCM, CCM1, and CCM2

Transients were also observed in each of the diodes in CCM 1. The switching frequency remained at a fixed 56.5 $\mathrm{kHz}$ and the output voltage was $10.5 \mathrm{~V}$. The waveforms can be seen in Figure 9. A current spike can be seen on the rise of each of the sinusoidal waveform. Immediately following the spike ringing oscillation can be seen. 


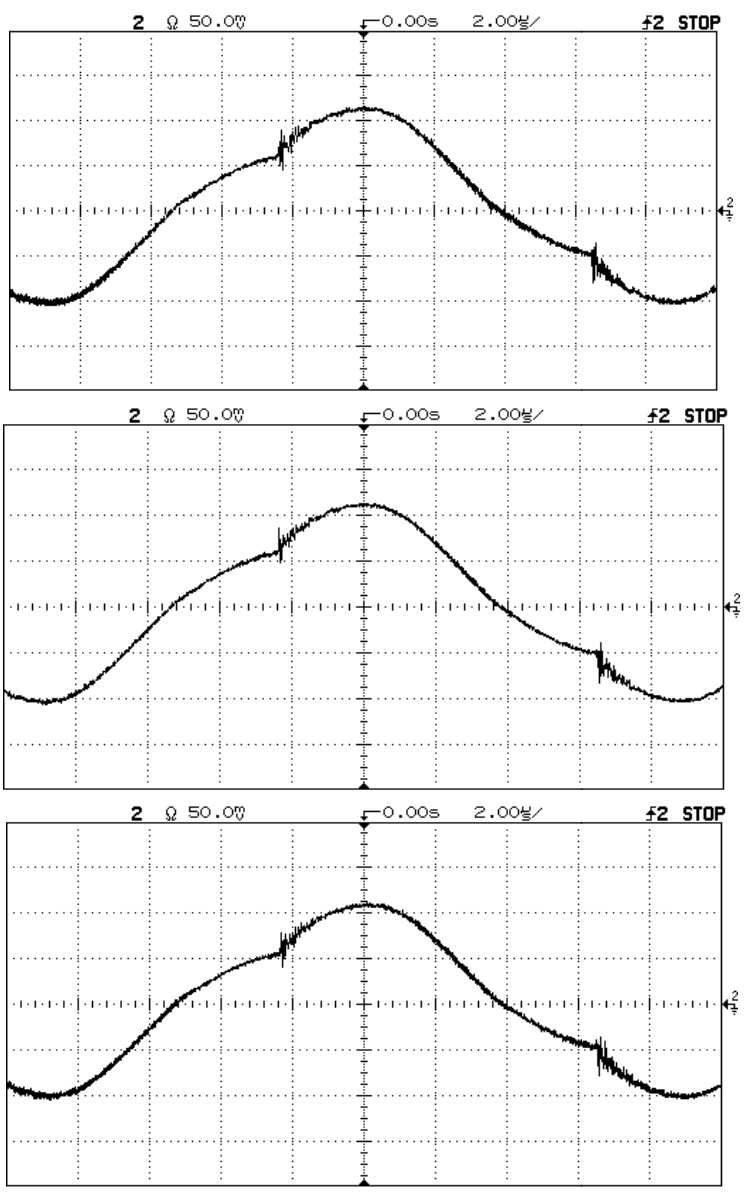

Figure 9. Switching transients in CCM1 using p-n diode (top), Schottky diode (middle), and SBR diode (bottom).

Overall, the results from simulation and hardware measurements show that the Schottky diode appears to have the best performance in CCM 1. The efficiency of the Schottky was higher throughout this conduction mode. It is difficult to say how significant this difference is in that the efficiencies of the three diodes stayed within $0.5 \%$. This is due to the fact that Schottky has the shortest reverse recovery time which is a critical parameter in the circuits operation in CCM 1. Since the diodes turn on without ZCS and ZVS, switching losses are reduced by a shorter reverse recovery time. In CCM 2 the Schottky efficiency was average and was actually lower in DCM. This is expected since the reverse recovery time is not an important parameter when ZCS or ZVS occurs.

The SBR seems to be the best suited for DCM and CCM 2. The efficiency in these modes outperformed those of Schottky and p-n diode. It also showed that SBR is better suited at higher loads and lower frequencies. The better performance at lower frequencies could indicate that it has lower conduction losses. The results in CCM 1 may indicate that the reverse recovery time is not superior as can be seen by the manufacturer's information in Table 2 . They also may indicate that the significance of the reverse recovery time is not as much as was thought.
The transient response of all three diodes can be seen in Figure 9. The SBR appears to have the least amount of transient behavior followed by the Schottky and p-n diode. This was expected by the manufacturer's claims. However, the waveforms for the SBR and Schottky are nearly identical and their differences may be a result of measurement inaccuracies. It is apparent that the $p$-n diode has the worst transient behavior.

Simulation predicted that at heavier loads the Schottky would outperform the other two diodes in CCM 1 and this was verified with hardware measurement. The simulation also predicted that the $\mathrm{p}$-n diode would outperform the others at lighter loads. This was not the cause in hardware measurement, but instead the Schottky was better. This could be a result of modeling inaccuracies. The simulation is only as good as its model and they are not $100 \%$ reliable.

\section{CONCLUSION}

The objective of this study was to compare the performance of the SBR with a Schottky and p-n diode. The results showed that the three diodes performed similarly and only differed from each other by $1 \%$. The SBR actually outperformed the Schottky and p-n diode in DCM and CCM 2 while the Schottky was the best choice for CCM 1. The higher performance of the SBR in DCM and CCM 2 is most likely attributed to its low forward voltage drop. This efficiency improvement in DCM and CCM 2 can be compared with a similar study [6] of a buck converter in which a more significant improvement was seen.

Oscillations were observed when the diodes changed states and the shoot-through transients of the SBR seemed to be better than the other two diodes. All three diodes had their respective advantages. The Schottky had its fast reverse recovery time, the $\mathrm{p}$-n diode had its low reverse leakage current, and the SBR had its smaller forward voltage drop and smoother transient response.

\section{REFERENCES}

[1]. C. Q. Lee, and S. Sooksatra, "Performance characteristics of the fullbridge zero voltage switching PWM resonant converter", IEEE Transactions on Industrial Electronics, vol. 38, 462-468. 1991.

[2]. H. Pinheiro, P. Jain, and G. Joos, "Performance characterization of two self-oscillating controllers for parallel resonant converters operating with unity input power factor", Power Electronics Specialists Conference, vol. 1, pp: 692-698. 1997.

[3]. M. Zaki, A. Bonsall, and I. Batarseh, "Performance characteristics for the series-parallel resonant converter", Southcon, pp: 573-577. 1994.

[4]. M . K. Kazimierczuk, and C. Wu, "Frequency-controlled seriesresonant converter with synchronous rectifier", IEEE Transactions on Aerospace and Electronic Systems, vol. 33, 939-948. 1997.

[5]. Vladimir, R., Ankoudinov, A. L., Taufik, "Performance Super Barrier Rectifier-A New Generation of Power Diode", IEEE Transactions on Industry Applications, pp: 234-237. 2008. 